\title{
A Fuzzy Logic Shared Steering Control Approach For Semi-Autonomous Vehicle
}

\author{
Ali Hamdan ${ }^{1}$, Reine Talj $^{1}$ and Véronique Cherfaoui ${ }^{1}$
}

\begin{abstract}
This paper deals with the shared lateral control between the driver and the automated system for the lane keeping purpose. The objective of this shared control is to ensure road safety and enhance driving performance. The control authority is realized using the blended shared control that permits the fusion of two inputs via a fusion parameter. To do that, a driver model is detailed to represent the human in the loop. The vehicle longitudinal and lateral movement are performed by an autonomous system based on Super Twisting Sliding Mode (STSM) control approach. A Fuzzy Logic Controller followed by a situation-based analysis block for the decision-making process are developed for the fusion parameter determination. Finally, the proposed shared control is validated on Matlab/Simulink for two case studies with a complete nonlinear model of the vehicle validated on "SCANeR Studio" (OKtal) professional simulator.
\end{abstract}

Keywords: semi-autonomous vehicle, human-machine cooperative control, cooperative driving, shared lateral control, Fuzzy logic decision-making.

\section{INTRODUCTION}

Safety is an important criterion that should be realized while driving on the road. Road accidents are caused by the human's errors in most cases. According to National Highway Traffic Safety Administration (NHTSA) statistics, these errors lead to $90 \%$ of road accidents [1]. In a critical situation, when the driver is tired or distracted, the integration of an Advanced Driving Assistance System (ADAS) helps him to prevent this dangerous situation. There are many examples of ADAS systems, like ESP, ABS, and lane keeping assist systems. The latter can help the driver to stay within the lane and warn him when he is crossing the boundaries. These systems ensure safety and performance of driving tasks. However, these systems are dedicated for specific tasks, in some precise driving situations, and their functionalities are limited. Hence, full autonomous vehicles are taking an important attention in the researches and automotive industry where several tasks of ADAS systems are done by an autonomous system itself. But, the realization and commercialization of these autonomous systems need more time for many reasons: high cost of hardware and software, safety on the road, robustness of the system, test and validation of the system for any possible scenario on the public road, etc. The autonomous driving system is the result of different tasks: Environment perception, motion planning and vehicle control. However, many problems are caused by these modules, and will need more time to be fixed. Thus, a shared cooperative control is necessary to

\footnotetext{
${ }^{1}$ Université de Technologie de Compiègne, CNRS, Heudiasyc, CS 60 319, 60203 Compiègne, France. Email: ali.hamdan@hds. utc. fr
}

compensate the gap between the manual driving (level 0/1 and 2) and full autonomous driving (level 5) [2]. This new aspect of control in the automotive field aims to reduce the workload of the driver. It helps and assists him to prevent a critical situation when he is distracted or tired. In addition, interaction between the human and the autonomous system that keeps the driver in the loop, should compensate the limits and problems of autonomous systems and improve driving safety. Therefore, if a conflict occurs during driving, it will be reduced by this shared strategy of control.

In this work, we will focus on the shared lateral control for the lane keeping purpose. The vehicle is asked to realize a path following. Two case studies of driving are considered later to address this control strategy under different driving conditions. Many advanced studies have been presented in the literature to investigate shared control between driver and an automated system. In fact, these studies differ in the way how to manage the control authority between the two agents. A shared lateral control is presented in [3] to illustrate the authority of driving between the driver and the automatic system. A decision making algorithm using a weighting function related to the lateral error was used to manage this authority and switch between them. Similar approach is presented in [4] to realize the control authority through a blending parameter varying between 0 and 1 , adjusted manually or automatically depending on the driver's attention and situation. A first order filter applied to a coordination variable is used in [5], to determine the control authority depending on the product of the driver and assistance system torque. The authors in [6], [7] have been developing a shared steering control for lane keeping and obstacle avoidance. The control input of assistance system is computed considering the driver's activity which reflects his actions and intentions. The authors in [8] suppose that the steering torque provides the driver some degree of freedom to control his vehicle. For that, a shared control has been developed for the emergency obstacle avoidance, where the assistance torque is multiplied by a weight coefficient $\omega$ varying between 0 and 1 to do the control allocation. However, the conflict between the driver and the steering assistance increases with the increasing of parameter $\omega$. An assistance steer-by-wire system is presented in [9], where the fusion of two steering inputs is performed considering the availability of the driver via monitoring system. Another lane keeping assistance system is used in [10] based on the fuzzy Takagi-Sugeno control method. Finally, many factors related to safety and driver (avoidance of lane departure, 
prevention of high acceleration and excessive steering) are taken into account when calculating the fusion parameter to deal with the tire blowout on the road [11]. Other studies consider the driver's steering in the trajectory planning, where the desired trajectory of the system is adjusted to meet the driver's intention [12].

All these interesting studies have motivated us to develop a shared control for the lane keeping objective using steer-by-wire system. However, this method can be adapted to consider the tracking of a local trajectory with more complex maneuvers (overtaking, collision avoidance...). Thus, in our present work, a new shared lateral control is developed to manage the control authority between driver and autonomous system in order to reduce the conflict and promote road safety. The novelty of this work is illustrated in the computation of the fusion parameter. A Fuzzy Logic Controller (FLC) associated to a situation-based Analysis Block are used to determine the fusion parameter according to many dynamics criteria such as: the lateral deviation error, the conflict of the automated steering angle and the driver's confidence. Then, the proposed approach is tested for two case studies to demonstrate the effectiveness of this method to ensure safety and improve driving performance.

The paper structure is as follows: in Section II a driver model reformulation is provided to represent the driver in the loop. Then, a longitudinal and lateral controller for autonomous system are exposed based on the the Supertwisting sliding mode (STSM) control approach, to follow the desired trajectory at the desired velocity. A full description of the proposed shared lateral control is introduced in Section III. The shared lateral control authority is detailed to blend the two inputs. To do that, A fuzzy logic controller and a situation-based analysis block are developed to determine the fusion parameter considering the two case studies. In Section IV the two case studies, simulation results and analysis are described. The validation of the proposed shared control is done on Matlab/Simulink with a complete nonlinear model of the vehicle, validated on "SCANeR Studio" (OKtal) simulator. Finally, the conclusions and the perspectives for future work are given in Section V.

\section{SHARED STEERING ARCHITECTURE}

The block diagram of the shared control is given in the Fig. 1. Its main components are: the Driver Model, the Autonomous System (detailed in the following), and the shared lateral control (detailed in Section III).

\section{A. Driver Model}

The proposed human driver model for the lane keeping purpose in [13], [14] is used to represent the human's behavior in the loop. This model is based on the hypothesis that the driver uses the visual information to anticipate with the upcoming road changing, and the vehicle state's information (velocity, position, heading...) to position the vehicle on the road (Anticipatory and Compensatory action). The structure

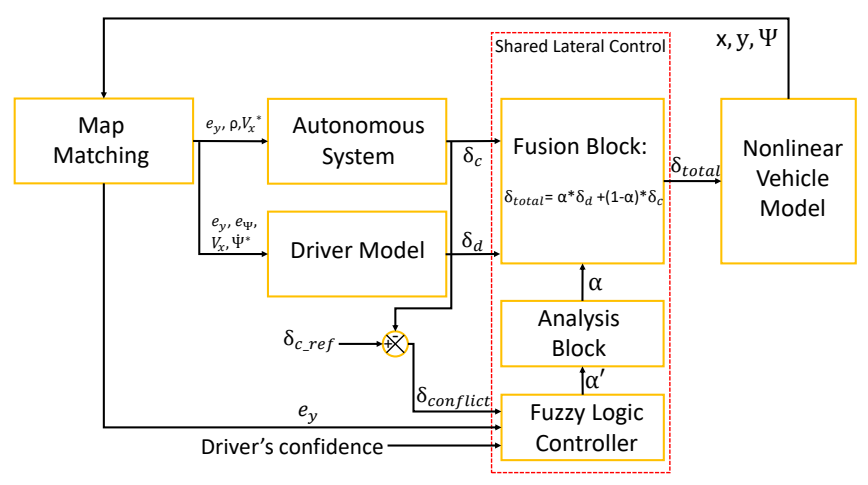

Fig. 1: Structure of the shared lateral control.

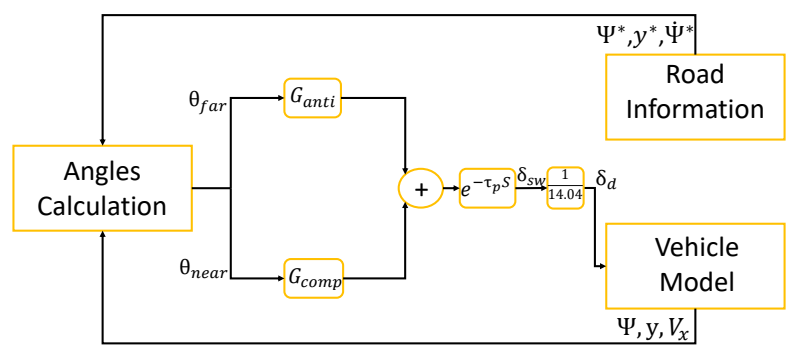

Fig. 2: Structure of the driver steering model.

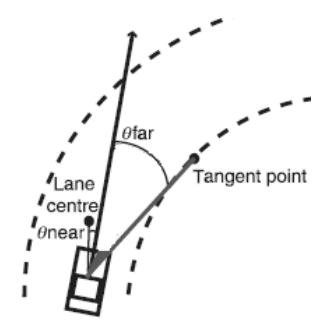

Fig. 3: $\theta_{\text {near }}$ and $\theta_{\text {far }}$ angles [14].

of the adopted driver model, used in this work, is given in Fig. 2. The calculation of angles' block that gives $\theta_{\text {near }}$ and $\theta_{\text {far }}$ angles is based on the definition in [14]. $\theta_{\text {near }}$ is calculated at a distance $l_{p}$ in front of the vehicle to maintain the vehicle's lateral position. It is function of the vehicle's heading error $e_{\psi}\left(e_{\psi}=\psi-\psi^{*}\right)$ and the lateral error $e_{y}\left(e_{y}=\right.$ $\left.y-y^{*}\right)$, where $\psi^{*}$ and $y^{*}$ are the desired heading angle and the lateral coordinate of the road respectively. $e_{\psi}$ and $e_{y}$ are calculated by a Map Matching block (see Fig. 1) that aims to localize the vehicle position on the reference map extracted from a "SCANeR Studio" scenario. $\theta_{f a r}$ is calculated as a tangent point (Fig. 3) at a far distance $D_{\text {far }}$ that depends on the curvature of the road. The far distance becomes constant when the driver drives on a curve road (usually between 10 and $20 \mathrm{~m}$ according to the radius of road curvature). It depends on the vehicle's velocity $V_{x}$ and the heading velocity 
of the road $\dot{\psi}^{*} . \theta_{\text {near }}$ and $\theta_{\text {far }}$ (see Fig. 3) are given as:

$$
\begin{gathered}
\theta_{\text {near }}=\frac{e_{y}}{l_{p}}-e_{\psi}, \\
\theta_{\text {far }}=\frac{D_{\text {far }}}{V_{x}} * \dot{\psi}^{*} .
\end{gathered}
$$

The visual information is composed of two parts: A compensatory part $G_{\text {comp }}$ that depends on $\theta_{\text {near }}$ to maintain a centerline position of the vehicle, and anticipatory part $G_{\text {anti }}$ which acts upon $\theta_{\text {far }}$ to consider the upcoming road curvature. $G_{\text {anti }}$ and $G_{\text {comp }}$ are given as:

$$
\begin{gathered}
G_{a n t i}=k_{p}, \\
G_{\text {comp }}=\frac{k_{c}}{V_{x}} \frac{T_{L} s+1}{T_{I} s+1},
\end{gathered}
$$

where $k_{p}$ is a positive constant parameter and $k_{c}$ represents the driver's cautiousness when he drives close to the lanes markers. $T_{L}$ and $T_{I}$ define the compensation frequency band and the compensation rate respectively. $s$ is the Laplace transformation variable. Finally, the visual processing delay module is represented by $e^{-\tau_{p} s}$. An approximation for the visual processing delay is given as:

$$
e^{-\tau_{p} s}=\frac{1-0.5 \tau_{p} s}{1+0.5 \tau_{p} s}
$$

where $\tau_{p}$ represents the time delay. Thus, the output of the adopted driver model is the steering wheel angle $\delta_{s w}$. Noting that the relation between the steering wheel angle $\delta_{s w}$ and the real angle of rotation of the front wheels $\delta_{d}$ is given as a proportional function:

$$
\delta_{d}=\frac{\delta_{s w}}{14.04}
$$

The values of the different parameters are given in Table I [13].

TABLE I: Driver model Parameters [13]

\begin{tabular}{|c|c|}
\hline Parameters & Values \\
\hline$l_{p} ; D_{\text {far }}$ & $2 ; 20$ \\
$k_{c} ; k_{p}$ & $20 ; 2.5$ \\
$T_{L}: T_{I}$ & $2 ; 0.5$ \\
$\tau_{p}$ & 0.04 \\
\hline
\end{tabular}

\section{B. Design of Longitudinal and Lateral Controllers for Au- tonomous System}

The autonomous system consists of longitudinal and lateral controllers based on the super-twisting sliding mode (STSM) algorithm, validated separately to realize a path following at the desired velocity. The main idea of the STSM is to define a sliding surface, representing the desired behavior of the system, where the dynamic states are forced to reach this surface during a finite time and remain on it. Consider the second order system given as:

$$
\dot{X}=f(X, t)+g(X, t) u(t)
$$

where $X=[x, \dot{x}]^{T} \in \mathfrak{R}^{2}$ is the state vector, $u$ is the control input, and $f, g$ are continuous functions. $X^{*}$ is the desired state of $X$ with $X^{*}=\left[x^{*}, \dot{x}^{*}\right]^{T} \in \mathfrak{R}^{2}$. The error vector is given by $E=X-X^{*}=[e, \dot{e}]^{T} \in \mathfrak{R}^{2}$ where $e=x-x^{*}$ and $\dot{e}=\dot{x}-\dot{x}^{*}$. Therefore, a sliding variable $s$ with relative degree $r=1$ w.r.t the control input, is defined as:

$$
s=\dot{e}+k e .
$$

The second order derivative of $s$ is:

$$
\ddot{s}(s, t)=\Phi(s, t)+\xi(s, t) \dot{u}(t)
$$

where $\Phi(s, t)$ and $\xi(s, t)$ are unknown bounded functions. The goal of the Super-Twisting algorithm is to enforce the sliding variable $s$ to converge to zero $(s=0)$ in finite time. Assume that there exist positive constants $S_{0}, b_{\min }, b_{\max }, C_{0}$, $U_{\max }$ verifying for all $X \in \mathfrak{R}^{n}$ and $|s(X, t)|<S_{0}$ :

$$
\left\{\begin{array}{l}
|u(t)| \leq U_{\max } \\
|\Phi(s, t)|<C_{0} \\
0<b_{\text {min }} \leq|\xi(s, t)| \leq b_{\text {max }}
\end{array}\right.
$$

Thus, the control input based on the Super-Twisting Sliding Mode algorithm [15] is given as:

$$
u(t)=u_{1}+u_{2}\left\{\begin{array}{l}
\left.\left.u_{1}=-\alpha_{1}|s|^{\tau} \operatorname{sign}(s), \tau \in\right] 0,0.5\right] \\
\dot{u}_{2}=-\alpha_{2} \operatorname{sign}(s)
\end{array}\right.
$$

$\alpha_{1}$ and $\alpha_{2}$ are positive gains. The following conditions guarantee the finite time convergence:

$$
\left\{\begin{array}{l}
\alpha_{1} \geq \sqrt{\frac{4 C_{0}\left(b_{\max } \alpha_{2}+C_{0}\right)}{b_{\min }^{2}\left(b_{\min } \alpha_{2}-C_{0}\right)}} \\
\alpha_{2}>\frac{C_{0}}{b_{\min }}
\end{array}\right.
$$

The convergence analysis is shown in [16].

The controller synthesis is based on a robotic formalism model presented in [17], [18] (more details in APPENDIX), that represent the coupling between the longitudinal and lateral dynamics. Based on this model, we choose the two sliding variables for the longitudinal and lateral controllers as follows:

$$
\begin{aligned}
& s_{1}=e_{V x}+\lambda_{x} \int e_{V x}, \lambda_{x}>0 \\
& s_{2}=\dot{e}_{y}+\lambda_{y} e_{y}, \quad \lambda_{y}>0
\end{aligned}
$$

where $\lambda_{x}$ and $\lambda_{y}$ are positive constants, and, $e_{V x}\left(e_{V x}=V_{x}\right.$ $\left.V_{x}^{*}\right)$ and $e_{y}$ are the vehicle longitudinal speed error and the lateral error respectively. The sliding variables $s_{1}$ and $s_{2}$ have a relative degree equal to one w.r.t the inputs respectively, the driving/braking torque $\Gamma_{c}$ for the longitudinal dynamics and the steering angle $\delta_{c}$ for the lateral dynamics. Thus, in order to converge these variables to zero and the controlled states follow the desired ones, and based on the above discussion, the torque and the steering angle control applied to the vehicle, are given by:

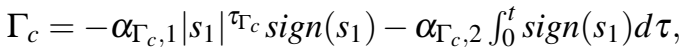

$$
\begin{aligned}
& \delta_{c}=u_{1}+u_{2}+\delta^{*}\left\{\begin{array}{l}
u_{1}=-\alpha_{\delta, 1}\left|s_{2}\right|_{\delta} \operatorname{sign}\left(s_{2}\right), \\
u_{2}=-\alpha_{\delta, 2} \int_{0}^{t} \operatorname{sign}\left(s_{2}\right) d \tau, \\
\delta^{*} \text { is the equivalent control input, }
\end{array}\right.
\end{aligned}
$$

where $\alpha_{\delta, i}$ and $\alpha_{\Gamma_{c}, i}$ with $i=[1,2]$, are positive constants satisfying the conditions in (12). $\tau_{\Gamma_{c}}$ and $\tau_{\delta}$ are constants in ] $0,0.5]$. The controller gains are given in [17]. Finally, $\delta^{*}$ is the equivalent control input, corresponding to the steering wheels angle at the equilibrium when $\dot{s}_{2}=0$. 


\section{DESIGN OF SHARED LATERAL CONTROL}

This section provides a full description of the proposed shared lateral control (see Fig. 1). The fusion approach based on the fuzzy logic controller and the situation-based analysis block are developed in the following for decision-making.

\section{A. Shared Lateral Control Authority}

The shared control authority between the driver and the autonomous system is performed by using the blended shared control [4] which allows the fusion of two inputs from each agent. This type of shared control is used for the systems where there is no mechanic connection between both inputs such as the steer-by-wire system. The familiar form of blending shared control is the blending using weight parameter (see. [11], [19]). The total blending control input is given as:

$$
\delta_{\text {total }}=\alpha * \delta_{d}+(1-\alpha) * \delta_{c},
$$

where $\delta_{\text {total }}, \delta_{d}$ and $\delta_{c}$ are the total control input, inputs of human driver and automated system respectively. $\alpha$ is the fusion parameter representing the influence proportion of each agent on the total input. $\alpha$ is bounded in $[0,1]$. The blending shared control permits a direct interaction between human driver and autonomous system. The fusion parameter $\alpha$ is calculated based on Fuzzy Logic Controller and a situation-based analysis block (Fig. 1) detailed later.

\section{B. Fuzzy Logic Controller (FLC)}

An intermediate fusion parameter $\alpha^{\prime}$ is determined by a fuzzy logic controller (FLC) as in Fig. 1. The situation-based analysis block analyzes the driving situation and gives the final value of fusion parameter $\alpha$ in order to promote driving safety and avoid dangerous situations. The fuzzy logic structure is given by the Fig. 4. The lateral error $e_{y}$, the conflict of the automated steering angle $\delta_{\text {conflict }}$ and the driver's confidence are applied to the fuzzy logic controller (FLC) as inputs, and the fuzzy parameter $\alpha^{\prime}$ is the output. Two scenarios are discussed and later addressed to demonstrate the effectiveness of this approach against different driving conditions. Using the FLC for the decision-making process for the cooperative control, makes the determination of the intermediate fusion parameter $\alpha^{\prime}$ simple, depending on the lateral error, the conflict of the automated steering angle $\delta_{\text {conflict }}$ and the driver's confidence. Note that the conflict of the automated steering angle $\delta_{\text {conflict }}$ is the difference between the steering wheel angle applied by the autonomous system $\left(\delta_{c}\right)$ and the reference steering angle $\left(\delta_{c-r e f}\right)$ on the desired trajectory given as:

$$
\delta_{\text {conflict }}=\left|\delta_{c-r e f}-\delta_{c}\right|,
$$

On the other hand, the driver's confidence can be determined in different ways depending on: driver's eyes observation, environment conditions, trajectory prediction, etc. However, the calculation of driver's confidence is not in the scope of this paper and it is considered as an input to the FLC fusion block. Three fuzzy sets are defined for the lateral

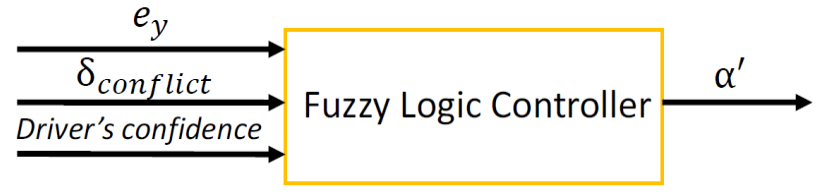

Fig. 4: The structure of fuzzy logic controller.

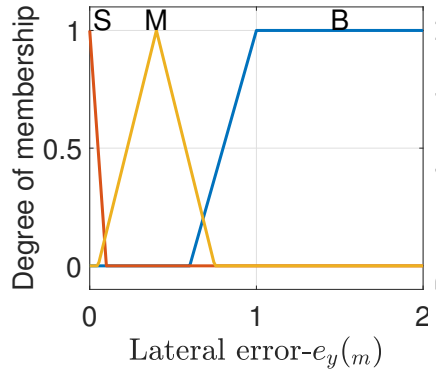

(a) Fuzzy sets - input 1

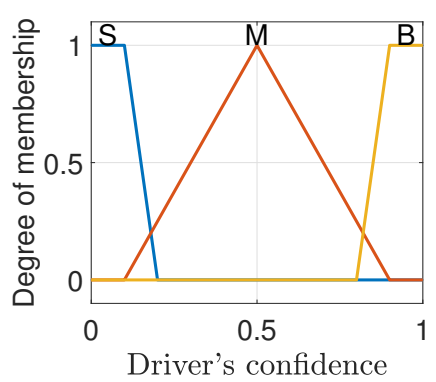

(c) Fuzzy sets - input 3

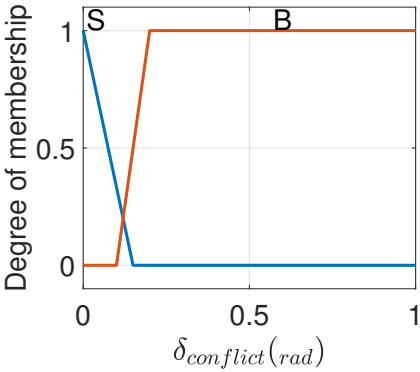

(b) Fuzzy sets - input 2

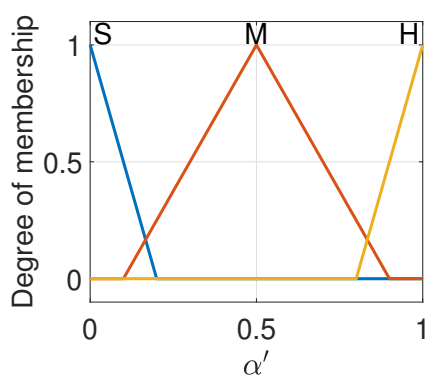

(d) Fuzzy sets - output
Fig. 5: Fuzzy sets of the inputs and the output

error $e_{y}:\{S($ Small $), M($ Medium $), B(B i g)\}$. Two fuzzy sets are defined for the conflict automated steering angle $\delta_{\text {conflict }}$ : $\{S($ Small $), B(B i g))\}$. Note that $\delta_{\text {conflict }}$ and the autonomous system's confidence are directly dependent. A small $\delta_{\text {conflict }}$ corresponds to a high autonomous system's confidence and vice-versa. Three fuzzy sets are defined for the driver's confidence: $\{S$ (Small), M(Medium $), B(B i g)\}$. And finally, three fuzzy sets are defined for the output parameter $\alpha^{\prime}$ : $\{S($ Small $), M($ Medium $), H($ High $)\}$.

The normalized Membership Functions (MFs) of fuzzification of the controller inputs and defuzzification of the controller output are respectively given in Figures. 5a, 5b, 5c and $5 \mathrm{~d}$. The values and the types of these fuzzy sets are chosen depending on the feedback and observation of simulation for different scenarios and a lot of experiments. To determine the fuzzy controller output $\alpha^{\prime}$ for the given fuzzy controller inputs $e_{y}, \delta_{\text {conflict }}$ and the driver's confidence, the decision matrix of the linguistic control rules is used. There are 18 rules (in total) where 15 are determined in the FLC to give the value $\alpha^{\prime}$, and the 3 remaining rules are detailed in the analysis block (see Fig. 1). So, the analysis block receives $\alpha^{\prime}$ and modifies it if necessary, depending on the driving situation. The final output of the analysis block is $\alpha$. Noting 
that $\alpha$ is equal to the output of FLC $\alpha^{\prime}$ if no check needed. The goal of using the analysis block with the FLC is to cover all the possibilities of driving situations that can occur while driving on the road. The rules of FLC are designed and presented in the Table II and Table III for $\delta_{\text {conflict }}$ small or big respectively. These fuzzy sets, membership functions, and the linguistic rules are usually determined based on an expert knowledge of the system by performing several simulations for different driving conditions. Finally, to defuzzify the result/output, the "Mamdani centroid fuzzy inference method" is used [20].

TABLE II: Rules of FLC when $\delta_{\text {conflict }}$ is $\mathrm{S}$

\begin{tabular}{|cc|c|c|c|}
\hline \multirow{2}{*}{$\alpha^{\prime}$} & \multicolumn{3}{|c|}{$e_{y}$} \\
& & $\mathbf{S}$ & $\mathbf{M}$ & $\mathbf{B}$ \\
\hline \multirow{3}{*}{ Driver's confidence } & $\mathbf{S}$ & $\mathrm{S}$ & $\mathrm{S}$ & $\mathbf{M}$ \\
\cline { 2 - 5 } & $\mathbf{M}$ & $\mathrm{S}$ & $\mathrm{S}$ & $\mathrm{M}$ \\
\cline { 2 - 5 } & $\mathbf{B}$ & $\mathrm{S}$ & $\mathrm{H}$ & check case \\
\hline
\end{tabular}

TABLE III: Rules of FLC when $\delta_{\text {conflict }}$ is B

\begin{tabular}{|cc|c|c|c|}
\hline \multicolumn{1}{|c|}{$\alpha^{\prime}$} & & \multicolumn{3}{c|}{$e_{y}$} \\
& & $\mathbf{S}$ & $\mathbf{M}$ & $\mathbf{B}$ \\
\hline \multirow{3}{*}{ Driver's confidence } & $\mathbf{S}$ & check case & $\mathbf{M}$ & check case \\
\cline { 2 - 5 } & $\mathbf{M}$ & $\mathrm{H}$ & $\mathrm{H}$ & $\mathrm{H}$ \\
\cline { 2 - 5 } & $\mathbf{B}$ & $\mathrm{H}$ & $\mathrm{H}$ & $\mathrm{H}$ \\
\hline
\end{tabular}

\section{Situation-Based Analysis Block}

In order to check the remaining 3 cases, an algorithm (given below) is developed in the analysis block to cover the possible driving situations that cannot be resolved appropriately by the FLC approach.

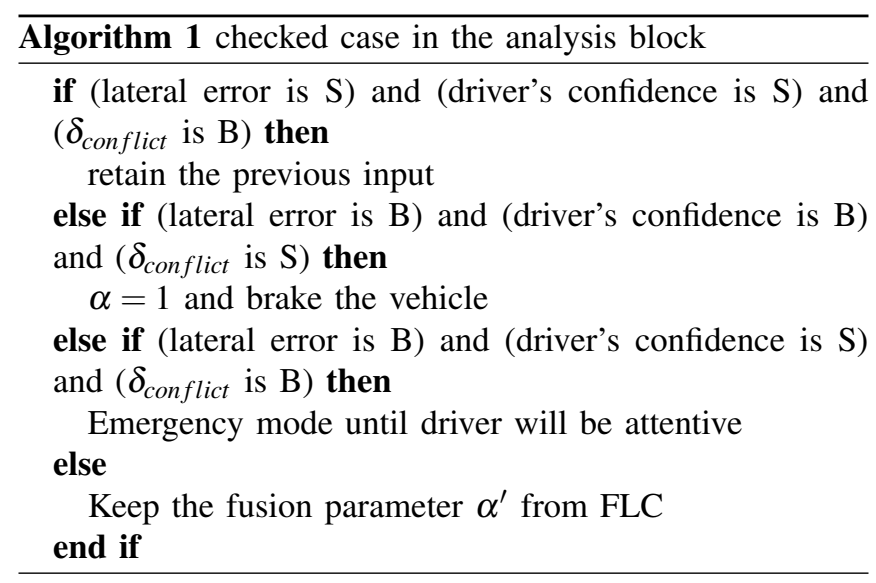

\section{VALIDATION OF THE APPROACH}

In this section, the developed shared lateral control is validated on Matlab/Simulink with a complete nonlinear model of the vehicle, validated on "SCANeR Studio" (OKtal) simulator. The vehicle is asked to follow the desired trajectory given in the Fig. 6, of a track extracted from a "SCANeR Studio" scenario. A control authority decision is generated by the FLC and the analysis block in order to determine the fusion parameter and then the leader of the vehicle for each case. The results are presented to show the adaptation and functionality of FLC and analysis block in each case.

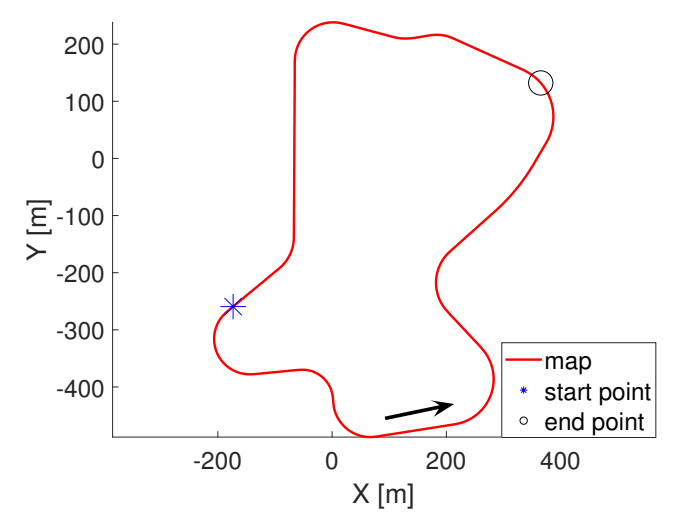

Fig. 6: Scaner Studio: Map of the test track

\section{A. Case Study Definition}

In this subsection, the case studies are detailed to test and validate the proposed shared lateral control for different driving situations. Indeed, two case studies are presented for the same scenario to show the adaptation and functionality of the proposed approach in each case:

- Case 1: This case is defined to show functionalities managed by the FLC. The developed controller aims to follow the desired trajectory by reducing the lateral error, at a desired velocity. It is addressed to assist the driver and help him in a shared control mode (an example of Advanced driving assistance system ADAS). A human driver (presented by the driver model in the simulation) is kept in the loop to supervise the scene and interact if necessary. A sudden failures occur in the autonomous system's behavior at $9 s$ and $40 s$ respectively (see Fig. 8). The vehicle deviates from the centerline of the road and the driving situation is considered dangerous. A driving mode is defined in a way to give the total authority of control to the driver at $9 s$ even when the driver's confidence is Medium (0.5)(Fig. 9). In addition, at 40s, the driver takes the control action to compensate the second failure of the automated system and retain the driving stability. Noting that the driver is aware and attentive about the scene at $40 s$ to the rest of trajectory (full Driver's confidence $=1$, see Fig. 9). The importance of this case is to illustrate the effectiveness of our method in terms of smooth switching between the two agents and failure's detection on the autonomous system. So, the control authority is determined through the fuzzy logic controller to manage the driving situation.

- Case 2: This case is defined to show the functionality of the analysis block with the FLC. Two functionalities of the analysis block are tested in this case: An emergency mode (EM) situation where the confidence in the driver is lost (Fig. 13) and a failure occurs on the autonomous system at a defined time (Fig. 12). The vehicle decelerates to stop. The ON/OFF conditions of emergency mode are given in the Fig. 7. Note that $e_{y_{\min }}$ and $V_{x_{\min }}$ are the lower limits of the lateral error and the longitudinal speed respectively, that define a no critical 
driving situation. $\delta_{\text {threshold }}$ is a threshold from which we consider that is an error on the autonomous system and the conflict on the automated steering angle is big that means the autonomous system's confidence is low. The second mode keeps the previous input that realizes a small lateral error when there is no driver's confidence (driver's confidence $=0$ ) at the same time of autonomous system's failure (Fig. 12). The driver's confidence varies between [0;1] (Fig. 13).

Results will be addressed in the next subsection, including the fusion parameter $\alpha$ to show the shared control authority between the two agents for each case given below.

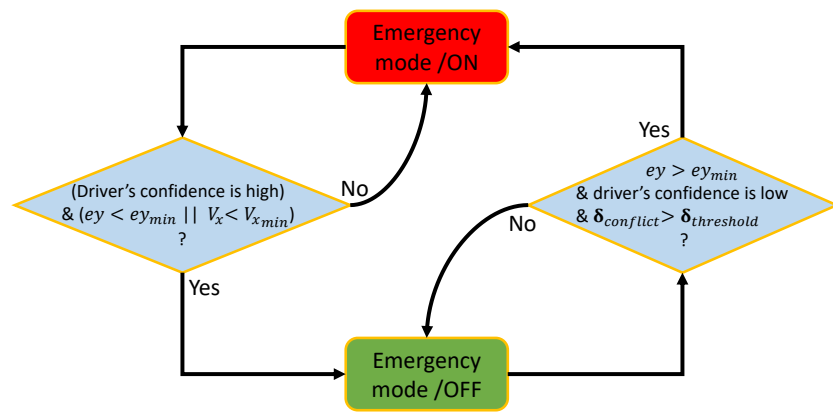

Fig. 7: Emergency mode ON/OFF

\section{B. Simulation Results}

For the case 1, errors are injected between $9 s$ and $23 s$ and between $40 s$ and $70 s$ respectively on the automated system (see Fig. 8). Remember that the human (presented by the driver model) is the supervisor of the scene who compensates for the errors that occurred in the behavior of the autonomous system.

The Fig. 8 shows the lateral error of: autonomous system with failure, manual driving mode and shared mode. As we can see, the shared mode diminishes the lateral error $(1.5 \mathrm{~m})$ caused by the autonomous system failure on the curvy road (see Fig. 11), by penalizing the last one and gives the authority of control to the driver. So, the driver takes the action of control at the beginning of the failure $9 s$, that is the decision of the FLC, until $23 s$, even though his confidence is medium (Fig. 9). The curve of lateral error in the shared mode is covered with the manual curve between $[9 s ; 23 s]$ (Fig. 8). Similar to the second failure, the driver acts on the vehicle at $40 s$ to $70 s$ in order to diminish the lateral error of autonomous system. A smooth switching is done between the two agents that makes the system stable. The shared control is done thanks to the fusion parameter $\alpha$ (Fig. 9) delivered by the FLC and analysis block modules. As shown in the Fig. 9, the value of $\alpha$ is equal to 0 expect the two intervals of time: $[9 s ; 23 s]$ and $[40 s ; 70 s]$. For the region where $\alpha=0$, the autonomous system is able to control the vehicle. However, for two intervals of time: $[9 s ; 23 s]$ and $[40 s ; 70 s]$, an unexpected error occurred on the autonomous system, illustrated by a conflict in the Fig. 10. At this time, the driver interacts by taking the action of driving to reduce the conflict of autonomous system and $\alpha$ increases from 0 to 1 . The STSM steering angle, the driver steering angle, the shared steering angle calculated by the fusion system and $\delta_{\text {conflict }}$ are given in the Fig. 10. And finally, Fig. 11 shows the longitudinal speed converging to the desired one through the STSM controller, the road curvature of the desired trajectory and finally, the actual lateral acceleration in the shared mode that demonstrate a stable and comfortable driving.

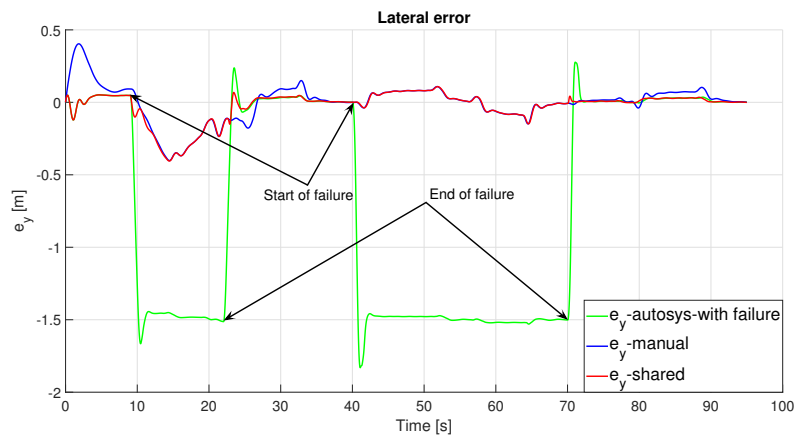

Fig. 8: The lateral error - case 1

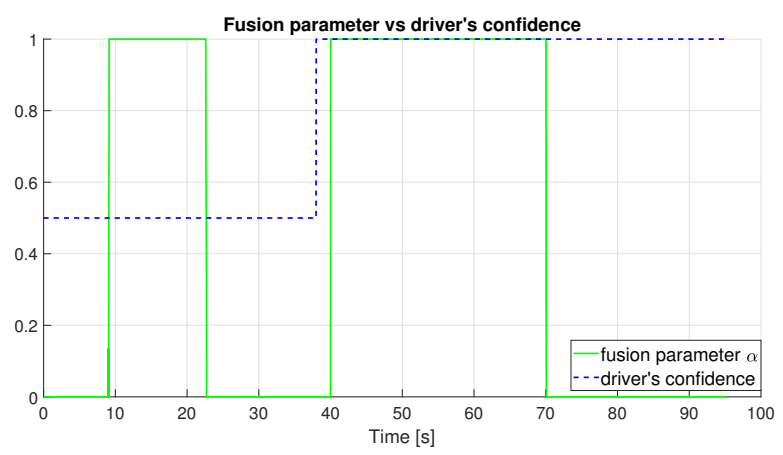

Fig. 9: The fusion parameter $\alpha$ - case 1

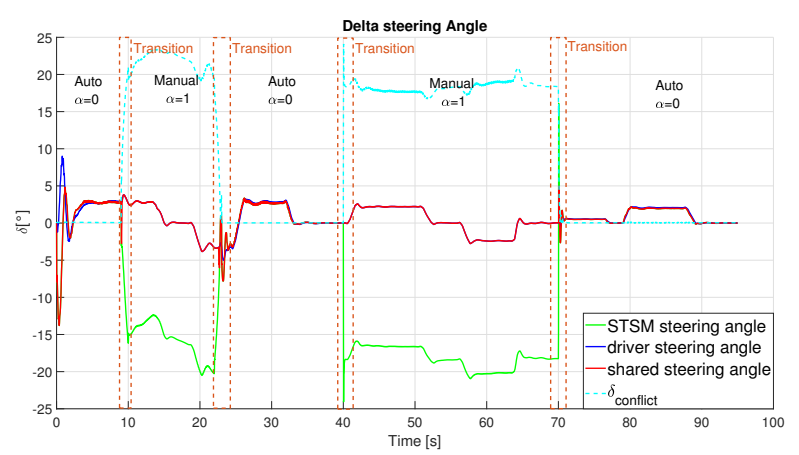

Fig. 10: The different steering wheel angles - case 1

Concerning case 2 the failure of autonomous system is injected at 40s until 70s (see Fig. 12) where there is no driver's confidence between $40 s$ and 50s (driver's confidence $=0$; see Fig. 13). An emergency mode is activated corresponding to $\alpha=1$ (Manual steering) while braking the vehicle that means the driver takes the driving task in the emergency mode situation until the vehicle is stopped (Fig. 13). Then, the 
driver is more attentive and careful to take the action of control between $50 s$ until 60s (driver's confidence $=1$; see Fig. 13). He is still acting on the vehicle's control between $60 s$ and $70 s$ even though his confidence decreased again to 0 (Fig. 13). Therefore, this mode is defined in the algorithm above which retains the previous input if it leads to a small lateral error, even if the confidence is small on this input. Fig. 12 shows the different lateral errors. The different values of fusion parameter $\alpha$ are given in Fig. 13, and Fig. 14 shows the different steering angles. The longitudinal speed, the road curvature of the desired trajectory, the driving/braking torque and the longitudinal and lateral acceleration of the shared mode are given in Fig. 15. As we can see in Fig. 15, when the emergency mode is activated, the vehicle starts the emergency mode by decreasing its velocity until the driver will be more attentive and will be able to drive the vehicle. And finally, the longitudinal speed starts to increase at $50 s$, ending the emergency mode. Noting that driving is comfortable for the overall trajectory including the emergency situation that ensure a stable driving situation. Moreover, many different tests are done for several driving situations to validate the proposed approach based on the decision making of the FLC and the different functionalities of block analysis.
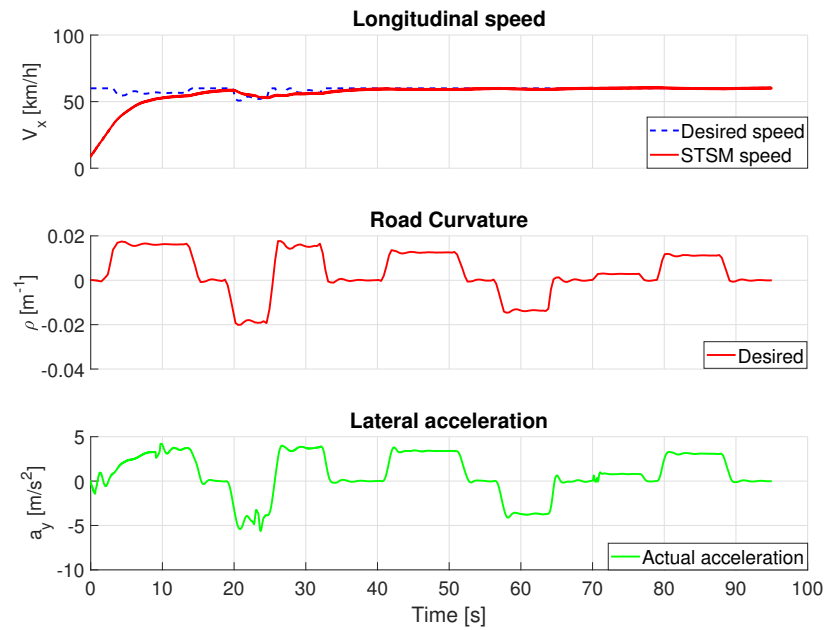

Fig. 11: Vehicle dynamic variables - case 1

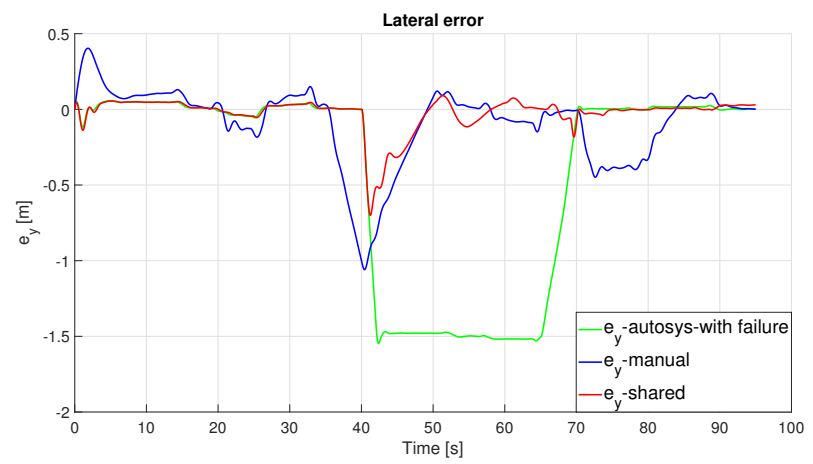

Fig. 12: The lateral error - case 2

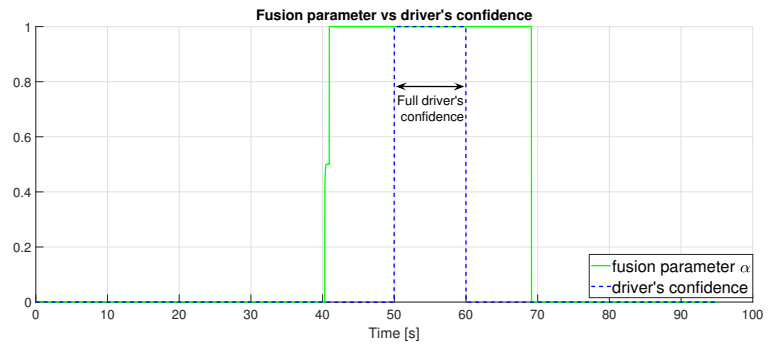

Fig. 13: The fusion parameter $\alpha$ - case 2

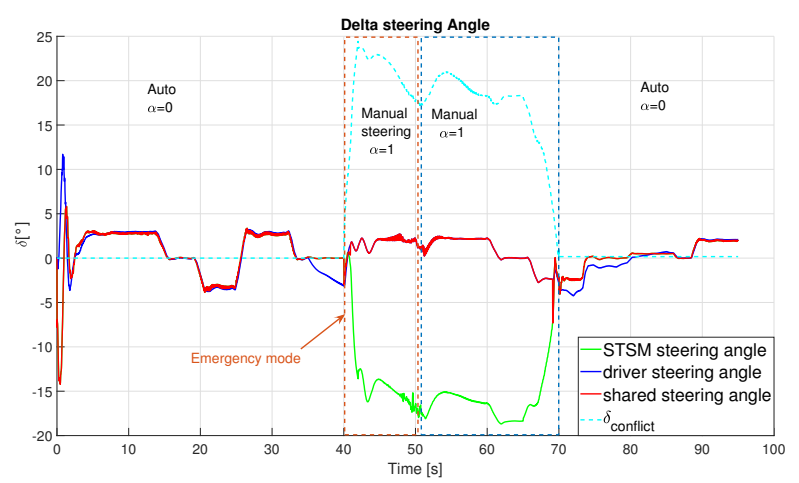

Fig. 14: The different steering wheel angles - case 2

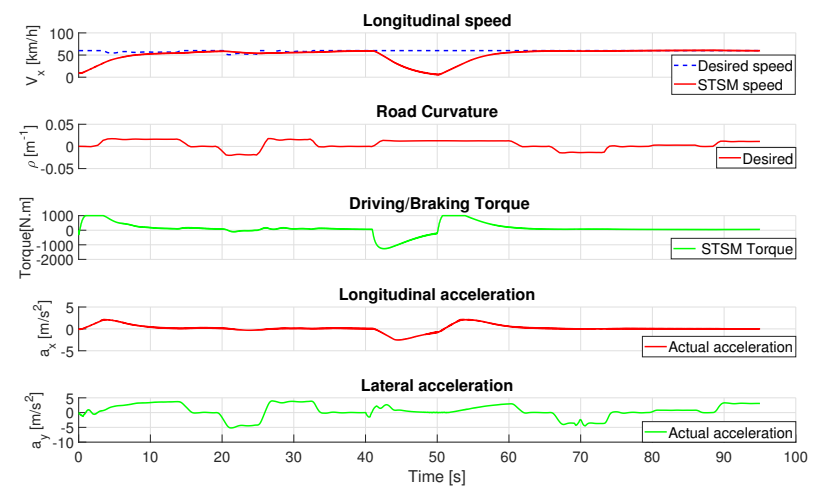

Fig. 15: Vehicle dynamic variables - case 2

\section{Conclusion And Perspectives}

To conclude, in this paper a shared lateral control has been developed to improve the system's performance and enhance driving safety. This cooperative control is done by using blended shared control, where a fuzzy logic controller and a situation-based analysis block are presented to determine the decision making authority. The proposed shared control is validated in Matlab/Simulink with a complete nonlinear model of the vehicle, validated on "SCANeR Studio" (OKtal) simulator. The validation of this shared control under different possible critical driving situations, is done in this work. In addition, the results show the effectiveness of the proposed approach to compensate the errors of autonomous system and prevent an undesirable driving situation. In the future work, we will consider other criteria to prove the effectiveness of 
the decision making process, and validate the approach on the "SCANeR Studio" simulator, connected to a hardware-inthe-loop steering system.

\section{APPENDIX}

The vehicle model is based on a robotic formalism model presented in [17], [18]. However, to simplify the controller design, some assumptions are given in [17]. With all assumptions, the reduced vehicle model used to establish control laws, can be written as:

$$
\begin{aligned}
& m_{e} \ddot{x}-m \dot{y} \dot{\psi}+L_{3} \dot{\psi}^{2}+F_{\text {aero }} \\
& +\delta\left(2 C_{\alpha_{f}} \delta-2 C_{\alpha_{f}} \frac{\dot{x}\left(\dot{y}+L_{f} \dot{\psi}\right)}{\dot{x}^{2}-\left(\frac{E}{2} \dot{\psi}\right)^{2}}\right)=g_{1} \\
& m \ddot{y}+m \dot{x} \dot{\psi}-L_{3} \ddot{\psi} \\
& +2 C_{\alpha_{f}} \frac{\dot{x}\left(\dot{y}+L_{f} \dot{\psi}\right)}{\dot{x}^{2}-\left(\frac{E}{2} \dot{\psi}\right)^{2}}+2 C_{\alpha_{r}} \frac{\dot{x}\left(\dot{y}-L_{r} \dot{\psi}\right)}{\dot{x}^{2}-\left(\frac{E}{2} \dot{\psi}\right)^{2}}=g_{2} \\
& I_{3} \ddot{\psi}+2 L_{f} C_{\alpha_{f}} \frac{\dot{x}\left(\dot{y}+L_{f} \dot{\psi}\right)}{\dot{x}^{2}-\left(\frac{E}{2} \dot{\psi}\right)^{2}} \\
& -2 L_{r} C_{\alpha_{r}} \frac{\left.\dot{x}\left(\dot{y}-L_{r} \dot{\psi}\right)\right)}{\dot{x}^{2}-\left(\frac{E}{2} \dot{\psi}\right)^{2}}-L_{3}(\ddot{y}+\dot{x} \dot{\psi})=g_{3}
\end{aligned}
$$

where $x$ and $y$ are the longitudinal and the lateral positions of the vehicle at its center of gravity (COG). $\psi$ is the yaw angle. $F_{\text {aero }}$ is the longitudinal aerodynamic force. $m_{e}, g_{1}, g_{2}$ and $g_{3}$ are given by:

$$
\begin{aligned}
& m_{e}=m+4 \frac{I_{w}}{R_{e f f}^{2}}, \\
& g_{1}=\frac{\tau_{w}}{R_{e f f}}, \\
& g_{2}=\left(2 C_{\alpha_{f}}-2 \frac{I_{w}}{R_{e f f}^{2}} \ddot{x}\right) \delta, \\
& g_{3}=L_{f} g_{2}+\left(-\frac{E}{2} C_{\alpha_{f}} \frac{E \dot{\psi}\left(\dot{y}+L_{f} \dot{\psi}\right)}{\dot{x}^{2}-\left(\frac{E}{2} \dot{\psi}\right)^{2}}\right) \delta .
\end{aligned}
$$

The control inputs to the vehicle are the steering wheel angle, $\delta$, and the Driving/Braking wheels torque $\tau_{w}$. More details about the vehicle parameters are given in [17].

\section{REFERENCES}

[1] R. Rajamani, Vehicle Dynamics and Control. Springer, 2012.

[2] F. M. Favarò, N. Nader, S. O. Eurich, M. Tripp, and N. Varadaraju, "Examining accident reports involving autonomous vehicles in california," PLoS one, vol. 12, no. 9, p. e0184952, 2017.

[3] C. Sentouh, S. Debernard, J.-C. Popieul, and F. Vanderhaegen, "Toward a shared lateral control between driver and steering assist controller," IFAC Proceedings Volumes, vol. 43, no. 13, pp. 404-409, 2010.

[4] F. Borroni and M. Tanelli, "A weighting approach to the shared-control of lateral vehicle dynamics," IFAC-PapersOnLine, vol. 51, no. 9, pp. 305-310, 2018.

[5] C. Sentouh, B. Soualmi, J.-C. Popieul, and S. Debernard, "Cooperative steering assist control system," in 2013 IEEE international conference on systems, man, and cybernetics. IEEE, 2013, pp. 941-946.

[6] A. Nguyen, C. Sentouh, and J.-C. Popieul, "Online adaptation of the authority level for shared lateral control of driver steering assist system using dynamic output feedback controller," in IECON 201541st Annual Conference of the IEEE Industrial Electronics Society. IEEE, 2015, pp. 003 767-003 772.

[7] A.-T. Nguyen, C. Sentouh, and J.-C. Popieul, "Driver-automation cooperative approach for shared steering control under multiple system constraints: Design and experiments," IEEE Transactions on Industrial Electronics, vol. 64, no. 5, pp. 3819-3830, 2016.
[8] K. Iwano, P. Raksincharoensak, and M. Nagai, "A study on shared control between the driver and an active steering control system in emergency obstacle avoidance situations," IFAC Proceedings Volumes, vol. 47, no. 3, pp. 6338-6343, 2014.

[9] G. Perozzi, C. Sentouh, J. Floris, and J.-C. Popieul, "On nonlinear control for lane keeping assist system in steer-by-wire road wheeled vehicles," 072020.

[10] B. Soualmi, C. Sentouh, J.-C. Popieul, and S. Debernard, "Fuzzy takagi-sugeno lq controller for a shared control of vehicle," in 2011 14th International IEEE Conference on Intelligent Transportation Systems (ITSC). IEEE, 2011, pp. 956-961.

[11] A. Li, Y. Chen, W.-C. Lin, and X. Du, "Shared steering control of tire blowout for ground vehicles," in 2020 American Control Conference (ACC). IEEE, 2020, pp. 4862-4867.

[12] M. A. Benloucif, A.-T. Nguyen, C. Sentouh, and J.-C. Popieul, "A new scheme for haptic shared lateral control in highway driving using trajectory planning," IFAC-PapersOnLine, vol. 50, no. 1, pp. 13834 $13840,2017$.

[13] L. Saleh, P. Chevrel, F. Mars, J.-F. Lafay, and F. Claveau, "Human-like cybernetic driver model for lane keeping," IFAC Proceedings Volumes, vol. 44, no. 1, pp. 4368-4373, 2011.

[14] F. Mars, L. Saleh, P. Chevrel, F. Claveau, and J.-F. Lafay, "Modeling the visual and motor control of steering with an eye to shared-control automation," in Proceedings of the human factors and ergonomics society annual meeting, vol. 55, no. 1. SAGE Publications Sage CA: Los Angeles, CA, 2011, pp. 1422-1426.

[15] J. Rivera, L. Garcia, C. Mora, J. J. Raygoza, and S. Ortega, "Supertwisting sliding mode in motion control systems," Sliding mode control, pp. 237-254, 2011.

[16] V. Utkin, "On convergence time and disturbance rejection of supertwisting control," IEEE Transactions on Automatic Control, vol. 58, no. $8,2013$.

[17] A. Chebly, R. Talj, and A. Charara, "Coupled longitudinal/lateral controllers for autonomous vehicles navigation, with experimental validation," Control Engineering Practice, vol. 88, pp. 79-96, 2019.

[18] A. Chebly, "Trajectory planning and tracking for autonomous vehicles navigation," Ph.D. dissertation, Université de Technologie de Compiègne, 2017.

[19] M. Li, H. Cao, X. Song, Y. Huang, J. Wang, and Z. Huang, "Shared control driver assistance system based on driving intention and situation assessment," IEEE Transactions on Industrial Informatics, vol. 14, no. 11, pp. 4982-4994, 2018.

[20] L. Reznik, Fuzzy controllers handbook: how to design them, how they work. Elsevier, 1997. 